\title{
Amélioration de la production laitière caprine par le croisement d'absorption dans une oasis du Sud tunisien
}

\author{
A. Gaddour ${ }^{1 *}$ S. Najari ${ }^{1}$ M. Ouni ${ }^{1}$
}

\begin{abstract}
Mots-clés
Caprin - Production laitière Croisement - Méthode d'amélioration génétique - Oasis - Tunisie.
\end{abstract}

\begin{abstract}
Résumé
Depuis 1980, un projet de croisement d'absorption de la chèvre locale Capra hircus, issue de la race Nubienne, par des races amélioratrices importées, a été mis en place dans une oasis d'une région aride de la Tunisie en vue d'améliorer la production laitière en élevage oasien intensif. L'analyse des performances laitières de certaines races caprines pures et des groupes génétiques croisés a été effectuée pour contribuer au choix d'une bonne race amélioratrice à utiliser dans le croisement d'absorption de la chèvre locale. Au total 1923 fiches de lactation de chèvres de races pures et croisées F1 et $\mathrm{F} 2$ ont servi à estimer les paramètres de production laitière : production totale traite $(\mathrm{kg})$, durée de lactation (jours) et moyenne journalière $(\mathrm{kg} / \mathrm{jour})$. Les variables quantitatives individuelles ont été soumises à des analyses de la variance (Anova) et des tests de comparaison de moyennes (Student, Newman et Keuls) ( $\alpha=5$ p. 100). Les résultats ont montré que la production laitière de la chèvre locale restait réduite même en conditions oasiennes intensives. Les performances des races importées ont été largement inférieures à celles réalisées dans leurs pays d'origine. Le croisement entre les races caprines a sensiblement amélioré la performance laitière de la chèvre locale. Alors que la production laitière moyenne a été de 133,5 kg par lactation chez la chèvre locale $(n=10)$, elle a été de $172,5 \mathrm{~kg}$ en première génération pour les trois types de croisements sans différence significative entre eux $(n=46)$, de 188,7 kg en deuxième génération pour les trois types de croisements $(n=46)$, et de $226,2 \mathrm{~kg}$ en deuxième génération pour le croisement avec la race Alpine $(\mathrm{n}=14)$.
\end{abstract}

1. Laboratoire de l'élevage et de la faune sauvage des zones arides et désertiques, Institut des régions arides, Médenine, Tunisie.

* Auteur pour la correspondance

Institut des régions arides, route Djorf $\mathrm{km} \mathrm{22,} 4119$ Médenine, Tunisie.

Tél : +21675633005; fax : +21675633006

E-mail : gaddour.omar@yahoo.fr essentiellement conduite en systèmes pastoraux et agropastoraux. Depuis sa domestication ancienne dans le croissant fertile, au sud-ouest de l'Asie, l'espèce caprine a pu s'adapter et maintenir sa présence dans les différents systèmes de productions animales du monde, et ce, surtout grâce aux différentes qualités des produits caprins $(2,6,17,21,33)$. Au niveau des oasis sahariennes, les chèvres jouent un rôle capital par leurs contributions diverses aux revenus de l'exploitation agricole $(13,22,27)$. Dans le système oasien, la chèvre profite d'une conduite intensifiée et peu sensible aux aléas climatiques qui prévalent en région aride. La faible productivité laitière de la chèvre locale en élevage pastoral est due à l'insuffisance des ressources naturelles, aux faibles capacités de production des systèmes de conduite traditionnelle (5) et à son potentiel génétique limité $(3,29)$. Pour remédier à ce dernier problème, des programmes de croisement de la chèvre 
locale sont une solution presque unique. En effet, la chèvre locale a génétiquement évolué pour renforcer ses capacités de rusticité et a été sélectionnée pour la production de viande ; par conséquent, la sélection peut difficilement produire rapidement des génotypes locaux laitiers $(25,29)$. A cet égard, un projet de croisement d'absorption de la chèvre locale a été conduit par l'Institut des régions arides de Médenine (Tunisie) pour produire des génotypes caprins aptes à valoriser les ressources oasiennes par une bonne production laitière. Pour atteindre cet objectif, des races performantes ont été importées et le croisement a débuté en 1980 dans la station de Chenchou.

C'est dans ce contexte que s'inscrit la présente étude qui porte sur l'analyse des données issues de croisement d'absorption de la chèvre locale par des races amélioratrices exotiques. Ces données, issues des années de contrôle des performances, permettent une comparaison zootechnique et économique des potentialités de ces nouveaux génotypes caprins dans les oasis. Les résultats doivent contribuer au choix de la meilleure race amélioratrice et du niveau de croisement à adopter, grâce à l'évaluation des potentialités laitières de la chèvre locale en conduite intensive et à l'étude de la possibilité d'amélioration de la production laitière caprine par le croisement.

\section{MATERIEL ET METHODES}

\section{Zone d'étude}

La présente étude a utilisé les données du contrôle laitier des différents génotypes caprins (races pures et croisées) élevés à la chèvrerie de l'Institut des régions arides de Médenine à la station de Chenchou située à $20 \mathrm{~km}$ de Gabès (sud de la Tunisie avec une latitude de $33^{\circ} 29^{\prime}$ nord et une longitude de $10^{\circ} 38^{\prime}$ est). La station est située dans l'étage bioclimatique aride inférieur ; la pluviométrie moyenne est autour de $188 \mathrm{~mm}$ par an, et plus de la moitié des précipitations sont observées aux mois de septembre, octobre et novembre. Le mois de janvier est le plus froid de l'année, avec une température moyenne de $10,7{ }^{\circ} \mathrm{C}$, alors que le mois d'août est le plus chaud avec une température de l'ordre de $27,3{ }^{\circ} \mathrm{C}(10)$.

\section{Matériel animal}

Le travail a porté sur l'étude des performances de production laitière individuelles de la population locale, des races amélioratrices et des génotypes issus de croisement d'absorption. Pour réaliser ce croisement d'absorption de la chèvre locale, trois races amélioratrices ont été choisies : la race Alpine connue pour ses hautes performances laitières, la race Murciana-Granadina, une race mixte à la fois laitière et prolifique, et la race Damasquine spécialisée dans la production de la viande (20). Dès 1980, des lots de chevrettes ont été importés de France, d'Espagne et de Chypre.

Le choix des races amélioratrices a été guidé par l'objectif du projet d'amélioration. En effet, les auteurs ont essayé d'utiliser des races du bassin méditerranéen possédant des aptitudes différentes, comme indiqué précédemment, avec la croissance des chevreaux liée à la production laitière et à la Murciana-Granadina pour améliorer la reproduction. Toutefois, l'usage d'autres races caprines pourrait être suggéré ; le nombre de races à utiliser est essentiellement fonction des ressources financières, humaines et matérielles. A cet égard, le coût élevé de l'exécution des schémas de croisement des animaux domestiques, et la difficulté technique de conduire et de suivre plusieurs génotypes en parallèle justifient cette restriction. En effet, les troupeaux de différents génotypes ont dû être conduits séparément toute l'année, ce qui a réduit le nombre de types génétiques à étudier. Le tableau I illustre les performances et les caractéristiques des races amélioratrices dans leurs pays d'origine.

\section{Chèvre locale}

Le cheptel caprin local constitue une population animale rustique possédant une large variabilité, tant au niveau de la morphologie qu'à celui des performances ; la moyenne de la production laitière par lactation est de 97,97 $\pm 87,63 \mathrm{~kg}$ (28). Cette population regroupe plusieurs types pigmentaires (32), probablement à cause de l'intégration de plus d'une race ou groupe génétique dans ses origines, à l'instar de la race Nubienne considérée disparue et diluée dans la population locale. D'ailleurs, la chèvre locale présente plusieurs caractéristiques communes avec la race Nubienne, mais des différences au niveau de la morphologie et des performances justifient l'usage de la notion de population locale (32).

La chèvre locale est un animal de petit format (hauteur $76 \mathrm{~cm}$ pour le mâle et $60 \mathrm{~cm}$ pour la femelle) avec un poids variable selon les ressources pastorales et les stades physiologiques. Au niveau des performances, la prolificité est en moyenne de 121 p. 100, et le poids des adultes mâles et femelles respectivement de l'ordre de 38 et $24 \mathrm{~kg}(32)$.

Généralement la chèvre locale est élevée pour produire la viande de chevreau, la production laitière étant réduite à une moyenne de 175 jours. La faiblesse de production laitière de la chèvre locale est en partie de nature génétique, toutefois, une grande variabilité des performances laitières est observée (28).

\section{Schéma de croisement}

Le croisement d'absorption de la chèvre locale consiste à réaliser un accouplement, à chaque génération, entre les femelles croisées (à la première étape, il s'agit de chèvres locales) avec des boucs de la race amélioratrice absorbante ; ce qui se traduit par une augmentation progressive du pourcentage des gènes de la race amélioratrice d'une génération à l'autre $(31,11)$, accompagnée d'une perte de rusticité. Le schéma de croisement est présenté dans la figure 1.

\section{Conduite des animaux et collecte des données}

Dès le démarrage du projet, les animaux ont été conduits en élevage intensif avec séparation des sexes et des groupes génétiques pour assurer le contrôle de la parenté. Les chèvres ont reçu une alimentation à l'auge composée de 2 à $3 \mathrm{~kg}$ de verdure produite en station (luzerne, orge en vert, sorgho), plus une quantité de foin qui variait de 0,5 à $1,2 \mathrm{~kg} /$ tête/jour, et environ $500 \mathrm{~g}$ de concentré pour toutes les chèvres. Elles ont reçu, en plus, une complémentation d'environ $300 \mathrm{~g}$ en fin de gestation et au début de la lactation.

\section{Tableau I}

Caractéristiques et performances des races amélioratrices dans leurs pays d'origine

\begin{tabular}{llcccc} 
Races & Origine & \multicolumn{2}{c}{ Poids adulte (kg) } & \multicolumn{2}{c}{ Lactation } \\
\cline { 3 - 3 } & & Mâle & Femelle & $\begin{array}{c}\text { Quantité } \\
\text { (kg) }\end{array}$ & $\begin{array}{c}\text { Durée } \\
\text { (jour) }\end{array}$ \\
\hline Alpine & France & 80 & 60 & 788 & 269 \\
Murciana & Espagne & 70 & 50 & 500 & 210 \\
Damasquine & Chypre & 80 & 60 & 200 & 90
\end{tabular}

Sources : FAO, 1991 ; Institut de l'élevage, site Web 


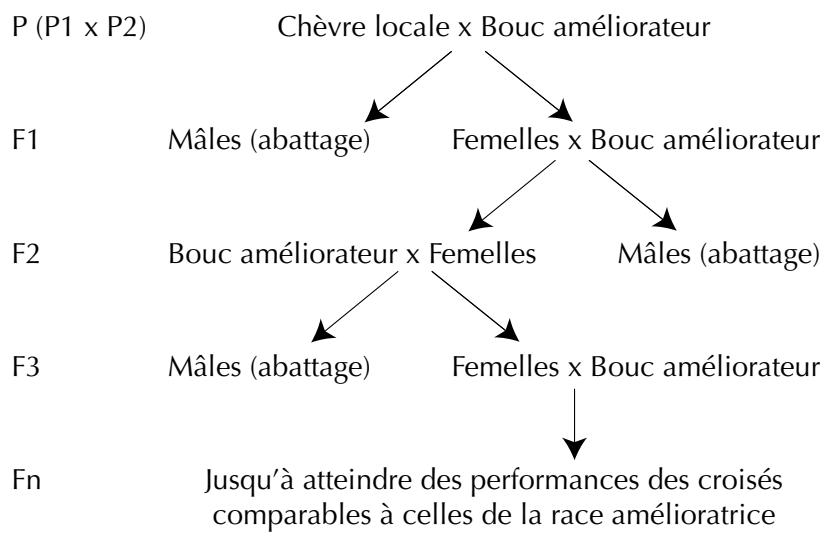

Figure 1 : schéma de croisement d'absorption de la chèvre locale.

Les animaux ont été identifiés dès la naissance. Un programme d'hygiène a été rigoureusement appliqué par l'équipe vétérinaire du projet. Les maladies parasitaires ont été les plus signalées. Par ailleurs, des problèmes de pneumonie ont sérieusement touché les jeunes et les adultes des races importées, faisant ainsi des dégâts considérables. La chèvrerie a été régulièrement badigeonnée d'insecticides et désinfectée avec des changements fréquents de la litière. Quant au programme prophylactique des animaux, les jeunes et les adultes ont été vaccinés contre les maladies contagieuses telles que la fièvre aphteuse et la brucellose (25). Egalement, une prévention contre l'entérotoxémie, et un traitement préventif contre les parasites internes et externes ont été appliqués deux fois par an. En dépit des soins vétérinaires, les taux de mortalité des chevreaux sont restés élevés.

Les contrôles laitiers ont été effectués périodiquement environ 12 heures après la séparation des chevreaux. La traite a été effectuée deux fois par jour le matin et le soir. Ce contrôle a débuté avec la première mise bas et a continué jusqu'au tarissement. Les chevreaux ont été sevrés après trois mois d'âge. La consommation de lait par les chevreaux n'a pas été évaluée.

Les données de base regroupaient l'information issue du contrôle laitier et celle relative à l'animal et aux mises bas. Pour chaque chèvre, la fiche annuelle contenait le groupe génétique, la campagne, la date de mise bas, la taille de la portée, les dates et les quantités de lait trait aux jours des contrôles.

A partir de ces fiches, trois variables ont été calculées pour chaque chèvre : la production totale de lait trait par lactation calculée par la formule de Fleischmann (20), la durée de la lactation et la moyenne de la production journalière traite.

\section{Analyses statistiques}

Les performances de production laitière ont été analysées pour les différents génotypes étudiés (la chèvre locale, les trois races amélioratrices et les groupes génétiques croisés).

\section{Analyse de la variance}

L'analyse de la variance pour les différentes performances a été effectuée selon le modèle statistique suivant :

$\mathrm{Y}_{\mathrm{ijkl}}=\mu+\mathrm{RA}_{\mathrm{i}}+\mathrm{AN}_{\mathrm{j}}+\mathrm{MN}_{\mathrm{k}}+\mathrm{RA}_{\mathrm{ij}} \mathrm{x} \mathrm{AN}_{\mathrm{ij}}+\mathrm{RA}_{\mathrm{ik}} \mathrm{x} \mathrm{MN}_{\mathrm{ik}}+$ $\mathrm{AN}_{\mathrm{jk}} \times \mathrm{MN}_{\mathrm{jk}}+\mathrm{e}_{\mathrm{ijkl}}$

où $\mathrm{Y}_{\mathrm{ijkl}}$ est la performance laitière analysée, $\mu$ la moyenne générale, $\mathrm{RA}_{\mathrm{i}}$ l'effet du génotype, $\mathrm{AN}_{\mathrm{j}}$ l'effet de l'année, $\mathrm{MN}_{\mathrm{k}}$ l'effet du mode de naissance, $\mathrm{RA}_{\mathrm{ij}} \mathrm{x} \mathrm{AN}_{\mathrm{ij}}$ l'effet de l'interaction race $\mathrm{x}$ année, $\mathrm{RA}_{\mathrm{ik}} \times \mathrm{MN}_{\mathrm{ik}}$ l'effet de l'interaction race $\mathrm{x}$ mode de naissance, $\mathrm{AN}_{\mathrm{jk}} \times \mathrm{MN}_{\mathrm{jk}}$ l'effet de l'interaction année $\mathrm{x}$ mode de naissance, $\mathrm{e}_{\mathrm{ijkl}}$ l'erreur résiduelle.

\section{Test de comparaison des moyennes}

La comparaison des performances moyennes par génotype et par facteur de variation a permis une évaluation des potentialités réalisées. La comparaison des moyennes des différents niveaux de chaque facteur étudié a été faite par le test de Student, Newman et Keuls $(\alpha=5$ p. 100). Les analyses des variances ainsi que les tests de comparaison des moyennes ont été effectués en appliquant le logiciel Spss (version 12.0).

\section{RESULTATS ET DISCUSSION}

\section{Sources de variation des performances laitières}

Les résultats de l'analyse de la variance des performances laitières sont consignés dans le tableau II. Ce tableau montre que le coefficient de détermination du modèle $\left(\mathrm{R}^{2}\right)$ a varié de 89 à 96 p. 100. Un effet très significatif $(\mathrm{P}<0,01)$ du facteur génotype sur la production totale par lactation et la moyenne de la production journalière a été décelé ; il a également été significatif $(\mathrm{P}<0,05)$ pour la durée de la lactation (33). Parmi les facteurs de milieu, l'effet de l'année a été très significatif sur la durée de la lactation, significatif sur la production totale et non significatif sur la moyenne journalière. La durée de la lactation en système intensif a varié essentiellement avec la conduite (28). Quant au facteur mode de naissance, son effet s'est avéré significatif $(\mathrm{P}<0,05)$ pour la moyenne journalière et la production totale, mais non significatif sur la durée de la lactation $(8,26)$.

Le tableau II montre que l'effet du génotype a été très significatif sur la production totale et la moyenne journalière de lait ; il a également été significatif sur la durée de la lactation. L'importance de l'effet des facteurs non génétiques paraît logique étant donné l'irrégularité des conditions environnementales dans les régions arides qui affectent les performances productives des chèvres. Ces effets peuvent être considérables, surtout chez les animaux des races importées. Il est en effet bien connu que le climat affecte les ressources alimentaires disponibles $(14,15,16,18,24)$. Toutefois, en élevage intensif, en l'absence d'une relation aussi directe entre l'alimentation et les facteurs climatiques, l'importance de l'action

\section{Tableau II}

Analyse de la variance des performances laitières des chèvres issues de croisement

\begin{tabular}{lccc} 
Facteurs/variables & $\begin{array}{c}\text { Production } \\
\text { totale } \\
(\mathbf{k g})\end{array}$ & $\begin{array}{c}\text { Moyenne } \\
\text { journalière } \\
\text { (kg/jour) }\end{array}$ & $\begin{array}{c}\text { Durée de } \\
\text { lactation } \\
\text { (jour) }\end{array}$ \\
\hline Génotype & $* *$ & $* *$ & $*$ \\
Année & $*$ & $\mathrm{NS}$ & $* *$ \\
Mode de naissance & $*$ & $*$ & $\mathrm{NS}$ \\
Génotype x année & $* *$ & $*$ & $* *$ \\
Génotype x mode N & $*$ & $\mathrm{NS}$ & $\mathrm{NS}$ \\
Année x mode N & $*$ & $\mathrm{NS}$ & $*$ \\
$\mathrm{R}^{2}(\%)$ & 89 & 94 & 96
\end{tabular}

* Significatif ; ** très significatif ; NS : non significatif

Mode $\mathrm{N}$ : mode de naissance $; \mathrm{R}^{2}$ : coefficient de détermination du modèle 
des facteurs non génétiques peut également trouver une explication à travers les composantes du milieu, comme la température, l'humidité et la photopériode.

\section{Effets du génotype}

Les performances moyennes de lactation des différents génotypes étudiés et leurs variations sont présentées dans le tableau III. Les données montrent que, parmi les races pures, la chèvre Alpine présentait les meilleures performances laitières avec une production moyenne de 244,4 kg qui s'étalait sur une durée de 132 jours de lactation, soit une production journalière traite moyenne de $1,85 \mathrm{~kg} / \mathrm{j}$. En seconde place, on trouve la race Murciana qui produisait $187,8 \mathrm{~kg}$ de production totale durant 156,5 jours de lactation, avec une production journalière de $1,2 \mathrm{~kg} / \mathrm{j}$. La race Damasquine vient ensuite avec une production totale de $177,1 \mathrm{~kg}$ pendant 145,1 jours, soit une moyenne journalière totale de $1,22 \mathrm{~kg} / \mathrm{j}$. La chèvre locale a enregistré la plus faible performance avec une production de $133,5 \mathrm{~kg}$ pendant 175,7 jours, soit $0,76 \mathrm{~kg}$ de lait par jour. Ces valeurs semblent être supérieures à celles mentionnées dans un rapport de la FAO (9), surtout pour les races Damasquine, Murciana et locale qui n'avaient donné respectivement qu'une production totale de $161,5,128,3$ et 48,2 kg. De même, ces valeurs sont inférieures à celles trouvées par Jalouali (20), qui mentionnait des productions totales de 276,34, 203,27 et $191,05 \mathrm{~kg}$, respectivement pour les races Alpine, Damasquine et Murciana dans les oasis du sud de la Tunisie.

Il faut également signaler que les performances réalisées par les races pures importées sont largement inférieures à celles réalisées par ces mêmes races dans leurs pays d'origine $(20,30)$. Au sein des génotypes issus de l'absorption, les chèvres croisées trois quart F2A (A x A1) ont montré une supériorité de leurs performances par rapport à celles des autres groupes, avec une production totale de 226,2 kg qui s'est étalée sur une période de 147,8 jours avec une moyenne de $1,53 \mathrm{~kg} / \mathrm{j}$. Les performances des croisées Alpines ont augmenté d'une génération à l'autre. En effet, la moyenne a varié de 1,17 à $1,53 \mathrm{~kg} / \mathrm{j}$ entre $\mathrm{F} 1 \mathrm{~A}$ et $\mathrm{F} 2 \mathrm{~A}$. Les chèvres croisées F1D (D x Lo) et F2D (D1 x Lo) ont présenté respectivement une production totale de 183,4 et $180,2 \mathrm{~kg}$, pendant une durée de 156,7 et 154 jours. Les croisées F2D (D1 x Lo) et F2M (M1 x Lo) ont eu une production totale plus faible, mais sans différence significative entre elles. Ces résultats montrent que le croisement d'absorption a permis d'améliorer les performances laitières de la chèvre locale.

\section{Interaction génotype $x$ milieu}

Dans les régions chaudes et à climat irrégulier, une importance particulière doit être accordée à la répétitivité de la réponse des génotypes à la grande marge de variation des composantes du milieu qui affectent la production (20). La régularité de la performance exprime la capacité des races à tamponner les fluctuations du milieu d'élevage et facilite la conduite (27).

L'analyse de la variance montre que les différents groupes génétiques étudiés manifestaient, en plus des différences au niveau des performances, un comportement variable en fonction de la variation des facteurs du milieu. La variation favorable ou défavorable d'un facteur correspond à une réponse variable pour chaque génotype.

Parmi les interactions révélant un effet significatif sur la production laitière, se trouvait celle représentant l'action combinée du génotype et de l'année, cette interaction montrant des aptitudes de production différentes des génotypes en fonction de l'année de production. Autrement dit, l'action d'une même campagne n'était pas ressentie de la même manière par les différents génotypes. Par ailleurs, l'interaction entre le génotype et le mode de mise bas

\section{Tableau III}

Performances de la lactation en fonction des génotypes

\begin{tabular}{|c|c|c|c|c|c|c|c|c|}
\hline \multirow[t]{2}{*}{$\begin{array}{l}\text { Groupe } \\
\text { génétique }\end{array}$} & \multirow[t]{2}{*}{$\begin{array}{l}\text { Nb. animaux } \\
\text { suivis }\end{array}$} & \multirow[t]{2}{*}{$\begin{array}{c}\mathrm{Nb} . \\
\text { performances }\end{array}$} & \multicolumn{2}{|c|}{$\begin{array}{l}\text { Production } \\
\text { totale (kg) }\end{array}$} & \multicolumn{2}{|c|}{$\begin{array}{l}\text { Moy./jour } \\
\text { (kg/jour) }\end{array}$} & \multicolumn{2}{|c|}{$\begin{array}{c}\text { Durée lactation } \\
\text { (jour) }\end{array}$} \\
\hline & & & Moy. & ET & Moy. & ET & Мoy. & ET \\
\hline Alpine (A) & 767 & 213 & $244,4^{\mathrm{a}}$ & 101,8 & $1,85^{a}$ & 0,52 & $132,1^{b}$ & 37,66 \\
\hline Damasquine (D) & 169 & 51 & $177,1^{a b}$ & 61,02 & $1,22^{a}$ & 0,33 & $145,1^{b}$ & 41,67 \\
\hline Murciana (M) & 148 & 46 & 187,8 ab & 75,13 & $1,20^{b}$ & 0,35 & $156,5^{a b}$ & 35,98 \\
\hline Locale & 148 & 10 & $133,5^{b}$ & 45,68 & $0,76^{\mathrm{c}}$ & 0,27 & $175,7^{a}$ & 26,72 \\
\hline $\mathrm{F} 1 \mathrm{~A}$ & 137 & 25 & $164,5 \mathrm{ab}$ & 95,27 & $1,17^{b}$ & 0,42 & $140,6^{b}$ & 39,38 \\
\hline$* \mathrm{~F} 2 \mathrm{~A}$ & 146 & 14 & $226,2^{a}$ & 115,7 & $1,53^{b}$ & 0,54 & $147,8^{b}$ & 18,12 \\
\hline F1D & 49 & 14 & 183,4 ab & 75,01 & $1,17^{b}$ & 0,36 & 56,8 ab & 26,47 \\
\hline$*$ F2D & 28 & 19 & 180,2 ab & 103,1 & $1,17^{b}$ & 0,46 & 154,0 ab & 40,83 \\
\hline $\mathrm{F} 1 \mathrm{M}$ & 15 & 7 & 179,4 ab & 84,04 & $1,12^{b}$ & 0,33 & $60,2 \mathrm{ab}$ & 35,72 \\
\hline${ }^{*} \mathrm{~F} 2 \mathrm{M}$ & 17 & 13 & $160,8^{a b}$ & 63,98 & $1,28^{b}$ & 0,41 & $125,6^{b}$ & 49,65 \\
\hline$* * \mathrm{~F} 1$ & 201 & 46 & 172,5 & 87,07 & 1,16 & 0,37 & 148,5 & 30 \\
\hline$* * *$ F2 & 191 & 46 & 188,7 & 107,1 & 1,31 & 0,43 & 144,1 & 35 \\
\hline
\end{tabular}

ET : écart-type

F1A, F2A : croisés Alpine x locale ; F1D, F2D : croisés Damasquine x locale ; F1M, F2M : croisés Murciana x locale

$a, b, c$ Groupes homogènes

* Désigne les descendants issus de croisement des femelles F1 avec les boucs de la race parentale

** F1 : moyenne de la première génération de croisement pour tous les génotypes

*** F2 : moyenne de la deuxième génération de croisement pour tous les génotypes 
reflétait une réponse différente des génotypes à un besoin en lait maternel croissant avec la taille de portée, illustré par le tableau IV. Plusieurs auteurs ont confirmé la relation entre la sécrétion lactée des chèvres et le niveau de stimulation relatif à la taille de portée $(12,20)$. Les chèvres produisent plus lorsqu'elles allaitent plusieurs chevreaux. Toutefois, les résultats relatifs à l'effet de l'interaction génotype $\mathrm{x}$ mode de mise bas ont montré que la réponse des chèvres à l'augmentation de la taille de la portée n'était pas homogène selon tous les génotypes. Entre autres, la variation des productions de chèvres allaitant des portées simples ou multiples a paru plus considérable chez la population locale. Ce fait témoigne de son instinct maternel prononcé.

\section{CONCLUSION}

L'étude de la production laitière de la chèvre locale et des génotypes caprins croisés a fourni un ensemble de paramètres indispensables pour évaluer les possibilités d'amélioration par le croisement en système oasien. En dépit de l'intensification, les performances laitières de la chèvre locale sont restées réduites, ceci témoignant des effets d'une sélection naturelle, sous les conditions de l'aridité, défavorable aux productions élevées, nutritionnellement coûteuses. La production laitière, qui reste le principal objectif de production caprine dans les oasis, a augmenté chez les génotypes croisés par rapport à celle de la chèvre locale. Par conséquent, le croisement peut être un moyen de résolution de la contrainte génétique pour la valorisation des ressources oasiennes, surtout lorsqu'une race laitière, comme l'Alpine, est utilisée.

L'analyse des performances laitières des génotypes caprins, sous des modes de conduite intensive, illustre l'important effet de l'environnement sur l'expression des potentialités génétiques laitières. D'ailleurs, les potentialités des races importées sont largement inférieures à celles réalisées dans leurs pays d'origine. L'intensification de la conduite n'annule pas les effets de l'environnement. Les résultats offrent de précieux outils pour la conception et pour l'application de plans d'améliorations

\section{Tableau IV}

Production laitière moyenne des chèvres de génotypes purs et croisés en fonction du mode de mise bas

\begin{tabular}{lrrrrr} 
Groupe génétique & \multicolumn{2}{c}{ Mise bas simple } & \multicolumn{2}{c}{ Mise bas multiple } \\
\cline { 2 - 3 } & & $\begin{array}{c}\text { Effectif } \\
\text { Production } \\
\text { totale (kg) }\end{array}$ & Effectif & $\begin{array}{c}\text { Production } \\
\text { totale (kg) }\end{array}$ \\
Locale & 4 & 94,82 & 6 & 159,33 \\
Alpine & 112 & 238,79 & 101 & 250,69 \\
Damasquine & 17 & 144,66 & 34 & 193,23 \\
Murciana & 26 & 169,05 & 20 & 212,07 \\
F1A & 15 & 145,64 & 10 & 192,85 \\
F2A & 11 & 234,07 & 3 & 197,4 \\
F1D & 5 & 125,28 & 9 & 215,7 \\
F2D & 8 & 131,05 & 11 & 215,91 \\
F1M & 4 & 196,65 & 3 & 156,33 \\
F2M & 8 & 177,3 & 5 & 134,46
\end{tabular}

F1A, F2A : croisés Alpine x locale ; F1D, F2D : croisés Damasquine x locale : F1M, F2M : croisés Murciana x locale génétiques appropriés en tenant compte des différents modes et systèmes d'élevage. Cependant la plus grande sensibilité des génotypes importés, comme en témoignent les cas de pneumonie constatés au cours de cette expérience, peut constituer un frein important à la mise en place de tels programmes.

D'autres schémas de croisements pourraient être testés en utilisant d'autres races caprines, comme la race Saanen, et en appliquant d'autres schémas de croisement. Par ailleurs, l'étude a concerné les performances de deux générations croisées seulement ; il reste important d'étudier les performances des générations plus avancées, l'amélioration ayant été meilleure à la deuxième génération d'absorption.

\section{BIBLIOGRAPHIE}

1. AMBROSOLI R., STASIO L., MAZZOCCO P., 1988. Content of alpha-S1-casein and coagulation properties in goat milk. J. Dairy Sci., 71: 24-28.

2. BAGHEL M.S., GUPTA M.P., 1979. Nitrogen distribution in goat milk. Indian J. Dairy Sci., 32: 340-342.

3. BENLEKHAL A., TAZI S., 1996. Les perspectives de développement de la filière lait de chèvre dans le bassin méditerranéen. Une réflexion collective appliquée au cas marocain. Rome, Italie, FAO, 45 p.

4. BOUlANGER A., GROSClAUDE F., MAHE M.F., 1984. Polymorphism of caprine (Capra hircus) alpha-s-1 and alpha-s-2-caseins. Genet. Sel. Evol., 16: 157-175.

5. CARUOLO E.V., 1974. Milk yield, composition, and somatic cells as a function of time of day in goats. Br. vet. J., 130: 380-387.

6. DE CREMOUX R., 1995. Relations entre les numérations cellulaires du lait et les infections mammaires chez la chèvre. Thèse Doct. Ecole nationale vétérinaire, université de Toulouse, France, $71 \mathrm{p}$.

7. DGPA, 2002. Statistiques de la direction de la production et du développement agricole. Paris, France, ministère de l'Agriculture, 45 p.

8. FABRE J.M., ROUSSE P., CONCORDET D., BERTHELOT X., 1990. Relations entre comptages cellulaires individuels et production en élevage bovin laitier dans le sud-ouest de la France : analyse critique des méthodes statistiques utilisées. Revue Méd. vét., 14 : 361-368.

9. FAO, 1991. Amélioration et développement des productions animales dans le Centre Sud Tunisien. Rapport technique. Rome, Italie, FAO, 35 p.

10. FLORET C., PONTANIER R., 1982. L'aridité en Tunisie présaharienne. Travaux et documents. Paris, France, Orstom, 544 p. ( $\left.{ }^{\circ} 150\right)$

11. GADDOUR A., 2005. Performances de croissance et de production laitière des groupes génétiques caprins issus d'un croisement d'absorption de la chèvre locale dans les oasis du Sud tunisien. Mastère Génétique et Bioressources, faculté des Sciences, Tunis, Tunisie, $73 \mathrm{p}$

12. GADDOUR A., NAJARI S., OUNI M., 2007. Dairy performances of the goat genetic groups in the southern Tunisia. Agricu. J., 2: 248-253

13. GADDOUR A., NAJARI S., OUNI M., 2007. Kid's growth of pure breeds and crossed caprine genotypes in the coastal oases of southern Tunisia. Res. J. Agron., 2: 51-58.

14. HAENLEIN G.F.W., 1980. Goats: Are they physiologically different from other domestic animals ? Goat Sheep Res., 1: 173-175.

15. HAENLEIN G.F.W., 1987. Cow and goat milk aren't the same, especially in somatic cell content. Dairy Goat J., 65: 806-826.

16. HAENLEIN G.F.W., 1991. Progress in sight for goat milk. United Caprine News (June): 34-35.

17. HAENLEIN G.F.W., 1992. Role of goat meat and milk in human nutrition. In: Proc. 5th International conference on goats, New Delhi, India, 1-8 March. New Delhi, India, ICAR, p. 575-580.

18. HINCKLEY L.S., 1991. Revision of the somatic cell count standard for goat milk. Dairy Food Environ. Sanit., 10: 548-549.

19. INSTITUT DE L'ELEVAGE. http://www.inst- elevage.asso.fr/html1/ article.php3?id_article $=9699$

20. JALOUALI S., 2000. Rentabilité du croisement d'absorption de la chèvre locale dans les oasis du Sud tunisien. Mémoire fin études, Ecole supérieure de Mograne, Tunisie, 134 p. 
21. JASH S., SINGH C., GUPTA A.K., 2001. Effect of enhanced prepartum concentrate feeding on kidding and lactation performance of stallfed crossbred goats. Indian J. Small Ruminants, 7: 19-24.

22. JEMALI M., VILLEMEOT M., 1996. L'expérience tunisienne en matière de filière lait caprin : le projet d'intensification de l'élevage caprin laitier dans les oasis tunisiens. Rome. Italie, FAO, 256 p.

23. JENNESS R., 1980. Composition and characteristics of goat milk. J Dairy SCi., 63: 1605-1630.

24. KALOGRIDOU-VASSILIADOU D., MANOLKIDIS K., TSIGOIDA A., 1992. Somatic cell counts in relation to infection status of the goat udder. J. Dairy Res., 59: 21-28.

25. LE GAL O., PLANCHENAULT D., 1993. Utilisation des races caprines exotiques dans les zones chaudes. Contraintes et intérêts. Maisons-Alfort, France, Cirad-emvt, $261 \mathrm{p}$.

26. MILLER R.H., EMANUELSSON U., PERSSON E., BROLUND L., PHILIPSSON J., FUNKE H., 1983. Relationships of milk somatic cell counts to daily milk yield and composition. Acta Agric. scand., 33: $203-$ 223.

27. NAJARI S., 2003. Goat production in the southern Tunisia. IGA Newsl. (Dec. 2003): 14-16.

28. NAJARI S., 2005. Caractérisation zootechnique et génétique d'une population caprine. Cas de la population caprine locale des régions arides tunisiennes. Thèse Doct. Etat, Institut national agronomique, Tunis, Tunisie, $214 \mathrm{p}$.
29. NAJARI S., BEN AHMED, 1996. Evaluation des résultats de croisement de la chèvre locale dans le Sud tunisien. Revue Rég. arides ( ${ }^{\circ}$ spécial) : 363-367.

30. NAJARI S., BEN HAMOUDA M., KHALDI G., 2000. Improvement of goat production in arid regions by the use of exotic breeds. In: Gruner L., Chabert Y., Eds, Nutrition and feeding strategies, Proc. 7th International conference on goats, Tours, France, 1999, p. 211-214.

31. NAJARI S., BEN HAMOUDA M., KHALDI G., 2002. Expression of the kid's genotypes in arid regions conditions. In: Elsen J.M., Ducroq $\mathrm{V}$., Eds, 7th Word congress on genetics applied to livestock production, Montpellier, France, 19-23 Aug. 2002, 33: 401-404.

32. NAJARI S., GADDOUR A., ABDENNEBI M., BEN HAMOUDA M., KHALDI G., 2006. Caractérisation morphologique de la population caprine locale des régions arides tunisiennes. Revue Rég. arides, 17 : 23-41.

33. POUTREL B., LERONDELLE C., 1983. Cell content of goat milk: California mastitis test, Coulter, and Fossomatic for predicting half infection. J. Dairy Res., 66: 2575-2579.

34. SALFER J.A., LINN J.G., OTTERBY D.E., HANSEN W.P., JOHNSON D.G., 1995. Early lactation responses of Holstein cows fed a rumen-inert fat prepartum, postpartum, or both. J. Dairy Sci., 78: 368-377.

Reçu le 16.09.2006, accepté le 12.03.2008

\section{Summary}

Gaddour A., Najari S., Ouni M. Improving Goat Dairy Production by Grading Up in an Oasis of South Tunisia

A local goat grading-up project has been on going since 1980 in an oasis of an arid region of Tunisia to improve goat milk production under intensive breeding conditions. The local goat (Capra hircus) originated from the Nubian breed and was graded up by imported breeds. A dairy performance survey was carried out on pure goat breeds and crossed genotypes to help select a good improved breed to use in local goat grading up. In total, 1923 data on the lactation of pure breeds, and F1 and F2 cross goats were used to estimate dairy production parameters: total milk production (kg), lactation length (days), and daily milk average ( $\mathrm{kg} /$ day). Individual quantitative variables were analyzed by ANOVA and by a post-hoc pairwise test (Student, Newman and Keuls) ( $\alpha=5 \%$ ). Results showed that the milk production of the local goat remained low even under intensive oasis conditions. The performances of imported breeds were much lower than those obtained in the country of origin. Crosses between goat breeds improved the milk performance of the local goat. The average milk production was $133.5 \mathrm{~kg}$ per lactation in the local goat $(\mathrm{n}=$ $10), 172.5 \mathrm{~kg}$ in the first generation for the three types of crosses without significant differences between them $(n=46)$, $188.7 \mathrm{~kg}$ in the second generation for the three types of crosses ( $\mathrm{n}=46)$, and $226.2 \mathrm{~kg}$ in the second generation for crosses with the Alpine breed $(n=14)$.

Keywords: Goat - Milk production - Crossbreeding - Breeding method - Oasis - Tunisia.

\section{Resumen}

Gaddour A., Najari S., Ouni M. Mejoría de la producción lechera caprina mediante el cruce de absorción en un oasis del Sur tunisino

A partir de 1980, se inició un proyecto de cruce de absorción de la cabra local Capra hircus, originaria de la raza Nubiana, con razas mejoradoras importadas, en un oasis de una región árida de Tunisia, con el fin de mejorar la producción lechera en una cría de oasis intensiva. Se efectuó el análisis de los rendimientos lecheros de ciertas razas caprinas puras y de grupos genéticos cruzados, con el fin de contribuir a la escogencia de una buena raza mejoradora para ser utilizada en el cruce de absorción de la cabra local. Se utilizaron en total 1923 fichas de lactación de cabras de razas puras y cruzadas F1 y F2 para estimar los parámetros de producción lechera: producción total al ordeño $(\mathrm{kg})$, duración de la lactación (días) y promedio por día (kg/día). Las variables cuantitativas individuales se sometieron al análisis de varianza (ANOVA) y a tests de comparación de promedios (Student, Newman y Keuls) $(\alpha=5 \%)$. Los resultados demostraron que la producción lechera de la cabra local se mantiene reducida incluso bajo condiciones intensivas en oasis. Los rendimientos de las cabras importadas fueron muy inferiores a aquellos realizados en sus países de origen. El cruce entre las razas caprinas mejoró sensiblemente el rendimiento lechero de la cabra local. Mientras que la producción lechera promedio fue de $133,5 \mathrm{~kg}$ por lactación para la cabra local $(\mathrm{n}=10)$, fue de $172,5 \mathrm{~kg}$ para la primera generación para los tres tipos de cruces sin diferencia significativa entre ellos $(n=46)$, de 188,7 kg para la segunda generación para los tres tipos de cruces $(n=46)$ y de $226,2 \mathrm{~kg}$ para la segunda generación para el cruce con la raza Alpina $(n=14)$.

Palabras clave: Caprino - Producción lechera - Cruziamento Método de mejoramiento genético - Oasis - Túnez. 